\title{
Polymer waveguides for THz QCL radiation delivery and filtering
}

\author{
Nazarov Maxim $^{1}$, A. Shilov ${ }^{2}$, Z. Margushev ${ }^{3}$, K. Bzheumikhov ${ }^{3}$, I. Ozheredov ${ }^{4}$, A. Angeluts ${ }^{4}$, \\ A. Sotsky ${ }^{2}$, A. Shkurinov ${ }^{4}$ \\ ${ }^{1}$ Kurchatov Institute National Research Center, Moscow, Russia, nazarovmax @ mail.ru \\ ${ }^{2}$ State Institution of Higher Education “A.A. Kuleshov State University”, Mogilev, Republic of Belarus \\ ${ }^{3}$ Institute of Computer Science and Problems of Regional Management of RAS, Nalchik, Russia \\ ${ }^{4}$ Department of Physics and International Laser Center, Lomonosov Moscow State University, Russia
}

The development of suitable waveguides with low attenuation will allow terahertz technologies to rise to a mass applications level. In the known reviews of perspective fibers for the $\mathrm{THz}$ range $[1,2]$ the necessary requirements of flexibility, single-mode regime and low attenuation are still not met simultaneously. For each case a compromise between the requested properties must be found. We demonstrate a possibility to meet all the requirements in a capillary waveguide structure in the case of the $\mathrm{THz}$ quantumcascade laser (QCL) radiation delivery. The tube lattice fiber (TLF) formed by one ring of the eight identical capillaries shown in Fig.1(c) is the best compromise for $\mathrm{THz}$ range. The advantages of this structure over other known types of waveguides are: a simple manufacturing process, the single-mode regime, small size and flexibility [3,4]. Discrete transparent spectral bands with sharp slopes inherent to this structure in some cases became an advantage of the TLF. We present manufacturing technology and theory allowing to optimize the TLF for the delivery of the narrow band QCL radiation at $f=3 \mathrm{THz}$ frequency.

The authors have extensive experience in the manufacturing of photonic crystal fibers from glass. Despite the difficulties in applying this technology to polymeric materials, we use original approaches to fabricate specified geometric parameters of waveguide structures in the multi-stage "stack and draw" process. As a result, a polypropylene flexible waveguides were manufactured in different configurations (Fig.1). Transmission properties of those TLF types are compared as well in the report as the most transparent polymer materials.
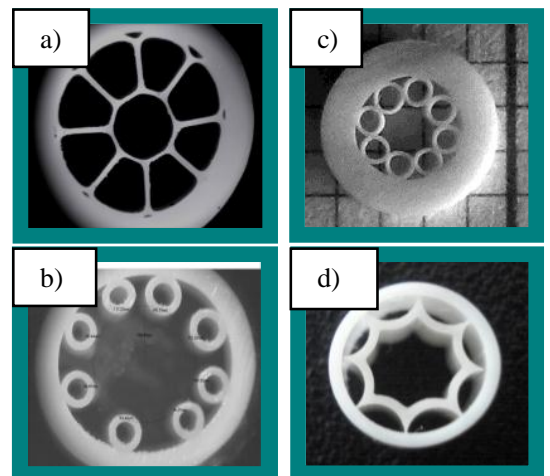

Fig. 1. Photo of manufactured polymer $\mathrm{THz}$ waveguides types

To create the most transparent and flexible waveguide for a fixed frequency, we studied in detail the influence of the core radius $R_{\text {core }}$ and capillary wall thickness $d$ (Fig.2(a)) on the shape and position of the transparency frequency windows. To characterize the fabricated TLF, it is necessary to take into account its imperfection. The wall thickness of each of the $8 \mathrm{ca}-$ pillaries in the "cladding" $d_{i}$ has a random deviation of $\delta d$ from the average value $\mathrm{d}$. With the improvement of manufacturing technology, the size dispersion $\delta d / d \approx 0.14$ should be minimized, it is now the limiting factor to decrease $d / R$ value and finally to decrease total TLF losses. The data in Fig.2(c) refer to polypropylene (PP) 8 capillary structure. Identical capillaries are tightly inserted into the tube cover. The ratio of the inner radius of the cover $\mathrm{R}$ to the outer radius of the capillaries $r$ is given as follows: $r=R /(1+1 / \sin (\pi / 8))$, $R_{\text {core }}=R-2 R /(1+1 / \sin (\pi / 8))=0.4465 \mathrm{R}$ Here $R$, $r$ and $R_{\text {core }}$ are explained on Fig. 2a). Note that number of capillaries can differ from 8 .

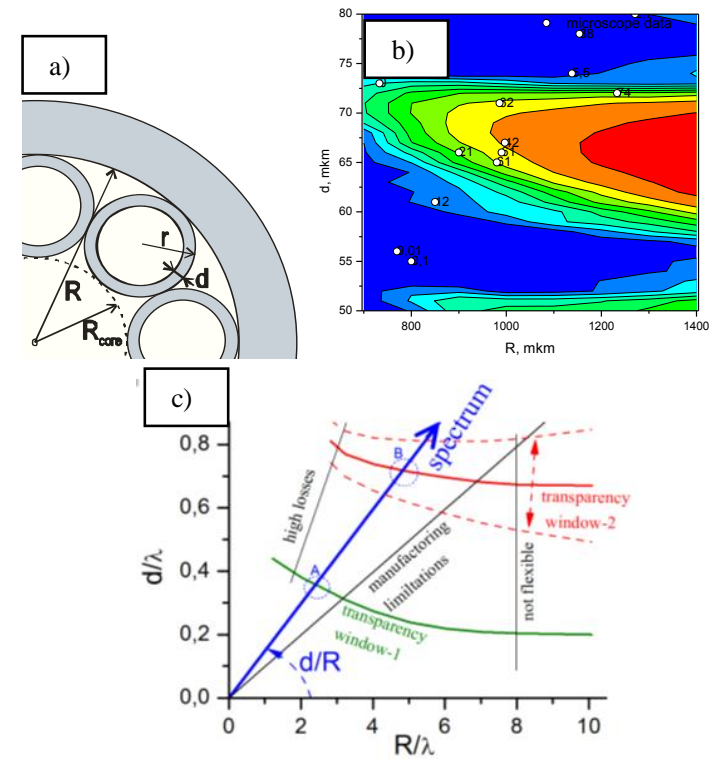

Fig. 2. a) TLF sizes, b) calculated (color area) and measured (circles) $30 \mathrm{~cm}$ TLF transmittance (in \%) vs $d$ and $R, \mathrm{c}$ ) Optimization strategy and physical limitations for 8 capillary dielectric $\mathrm{THz}$ waveguide. Optimal wall thickness $d v s$ air core radius $R$ for first two transparency windows, both axes are normalized to the wavelength $\lambda$. Markers $A$ and $B$ indicate a particular parameters set.

The used TLF structure is optimized by two most important values - the wall thickness $d$ (which determines the position of the transmission windows) and the core radius $R$, (affecting attenuation value, flexibility and modal regime). All structure proportions are given by the ratio $d / R$ of the initial tubes, that indi- 
cates how thick the wall relative to other TLF sizes is. In fact the maximum achievable transparency of TLF is determined by this ratio, it should be as low as possible till wall quality and TLF flexibility are satisfactory- Fig 2c).

Considerations for optimal $d$ should account for a quasiperiodic dependence of losses versus $d$. The actual spectral position of each transparency window depends on the size of the waveguide core $R$. We choose the second transparency window as the most promising of the technically available ones. To account for the deviation from analytical estimation [4], special calculations were carried out using the methods developed in [Error! Bookmark not defined.]. The relationship between $R$ and $d$ for the center of the first and second transparency windows for a structure of 8 polypropylene (PP, $n=1.5$ ) capillaries are shown in Fig. 2c). Since polymers refraction are almost constant in THz range, for TLF analysis variation of $d$ is identical to variation of $1 / \lambda$, in $d / \lambda$ scale we obtain universal curve (Fig. 2c), some difference appear between $R / \lambda=$ const and $d / R=$ const cases for $d$ or $\lambda$ variation [Error! Bookmark not defined.].

\section{The measurements}

To couple QCL radiation into the TLF we focused a free space radiation with a lens into a $1 \mathrm{~mm}$ spot on the input end - Fig. 3a). At the output end of the $30 \mathrm{~cm}$ long sample we measured the radiation by the Golay cell, THz camera or interferometer. For bending loss measurements we moved the output end of the waveguide together with the Golay cell.
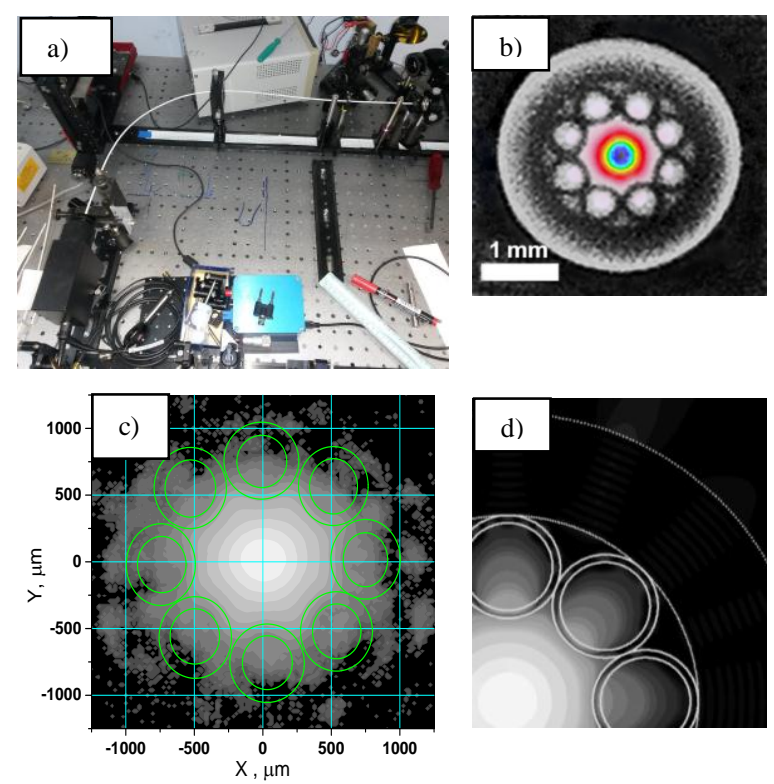

Fig.3 a) Experimental scheme photo, (b) and (c) $\mathrm{THz}$ field distribution of TLF mode cross-section in linear (b) and $\log (\mathrm{c})$ scales measured by $\mathrm{THz}$ camera. (d) Theory for fundamental mode field distribution in $\log$ scale.
More rigorously the shape and bandwidth of the transparency window can be measured in spectral domain by THz-TDS method for thicker walls and lower frequency - see Fig.4, for the same $\delta d / d$ and $d / R$ average values. Using scalability of $d / \lambda$, one can compare frequency-domain spectra with wallthickness domain spectra. The transparency window bandwidth and shape for fixed values of $\delta d / d$ and $d / R$ are in good agreement with the theory. Note, that $d$ and $\lambda$ values indicated by markers $B$ " in Fig. 4 correspond to the second transparency window center indicated by marker $B$ in Fig.2(c), in all cases $d / \lambda \approx 0.75$.

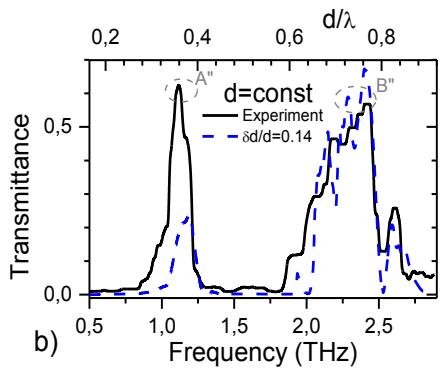

Fig. 4 Fixed $d=95 \mu \mathrm{m}$ and $R=600 \mu \mathrm{m}$, TDS transmittance spectra - solid magenta line; dashed blue line - theory for $\delta \mathrm{d} / \mathrm{d}=0.14$.

In conclusion, we have demonstrated a purely dielectric, flexible, single mode, narrowband waveguide for the $3 \mathrm{THz}$ frequency. In comparison with the previously known studies of TLF [5] we increased the used frequency from $0.3-1.5 \mathrm{THz}$ to $3 \mathrm{THz}$, decreased the transversal TLF size from $1 \mathrm{~cm}$ to $3 \mathrm{~mm}$. For filtering out undesirable frequencies of $\mathrm{THz}$ QCL radiation and selecting desired QCL frequency a set of waveguides can be used due to a steep slope of their transparency window. At the same time, the key application of the TLF seems to be a way to deliver single mode $\mathrm{THz}$ radiation to hardly accessible areas. A good agreement of the experimental data and theoretical calculations allows accounting for the existing technical limitations.

The work was supported by RFBR grants 18-5200040, 17-00-00270, 16-29-11789 and BRFFI grant F18R-143.

\section{References}

1. S. Atakaramians, S. Afshar, V. T. M. Monro, D. Abbott, Adv. Opt. Photon. 5, 169 (2013).

2. A. Bahr, B. P. Pal, G. P. Agrawal, R. K. Varshney, and M. A. Rahman, IEEE J. Sel. Topics Quantum Electron.22, 850015 (2016).

3. V. Setti, L. Vincetti, and A. Argyros, Opt. Express 21, 3388 (2013).

4 M.M. Nazarov, A.V. Shilov, K. A. Bzheumikhov, Z. Ch. Margushev, V. I. Sokolov, A. B. Sotsky, and A. P. Shkurinov, IEEE Trans. THz Sci. Technol. 8, 183 (2018).

5W. Lu, S. Lou, A. Argyros, IEEE J. of Sel.Top. In Quantum Electronics, 22, 4401607 (2016) 